Please do not remove this page

RMIT

UNIVERSITY

\title{
Imaging and quantum-efficiency measurement of chromium emitters in diamond
}

Castelletto, Stefania; Aharonovich, I; Gibson, Brant; Johnson, B.C; Prawer, Steven

https://researchrepository.rmit.edu.au/esploro/outputs/9921861944301341/filesAndLinks?institution=61RMIT_INST\&index=null

Castelletto, S., Aharonovich, I., Gibson, B., Johnson, B. ., \& Prawer, S. (2010). Imaging and quantum-efficiency measurement of chromium emitters in diamond. Physical Review Letters, 105(21), 217403-1-217403-217404. https://doi.org/10.1103/PhysRevLett.105.217403

Document Version: Published Version

Published Version: https://doi.org/10.1103/PhysRevLett.105.217403

Repository homepage: https://researchrepository.rmit.edu.au

(c) 2010 American Physical Society

Downloaded On 2023/04/27 00:54:41 +1000

Please do not remove this page 
Thank you for downloading this document from the RMIT Research Repository.

The RMIT Research Repository is an open access database showcasing the research outputs of RMIT University researchers.

RMIT Research Repository: http://researchbank.rmit.edu.au/

\section{Citation:}

Castelletto, S, Aharonovich, I, Gibson, B, Johnson, B and Prawer, S 2010, 'Imaging and quantum-efficiency measurement of chromium emitters in diamond', Physical Review Letters, vol. 105, no. 21, pp. 217403-1-217403-4.

See this record in the RMIT Research Repository at:

http://researchbank.rmit.edu.au/view/rmit:21950

Version: Published Version

Copyright Statement: (c) 2010 American Physical Society

Link to Published Version:

http://dx.doi.org/10.1103/PhysRevLett.105.217403 


\title{
Imaging and Quantum-Efficiency Measurement of Chromium Emitters in Diamond
}

\author{
S. Castelletto, ${ }^{*}$ I. Aharonovich, ${ }^{\dagger}$ B. C. Gibson, B. C. Johnson, and S. Prawer \\ ${ }^{1}$ School of Physics, The University of Melbourne, 3010 Victoria, Australia
}

(Received 2 July 2010; published 16 November 2010)

\begin{abstract}
We present direct imaging of the emission pattern of individual chromium-based single photon emitters in diamond and measure their quantum efficiency. By imaging the excited state transition dipole intensity distribution in the back focal plane of high numerical aperture objective, we determined its 3D orientation. Employing ion implantation techniques, the emitters were placed at various distances from the diamondair interface. By comparing the decay rates from the single chromium emitters at different depths in the diamond crystal, we measured an average quantum efficiency of $28 \%$.
\end{abstract}

PACS numbers: 78.47.jd, 78.55.- m, 78.67.Bf, 81.05.ug

Quantum efficiency is a fundamental property of any nanoscopic emitter since it dictates the ability to emit a photon once an excitation photon is absorbed. The quantum-efficiency $(\mathrm{QE})$ is defined as $\mathrm{QE}=k_{\mathrm{rad}}^{\infty} /\left(k_{\mathrm{nr}}+\right.$ $k_{\mathrm{rad}}^{\infty}$ ), where $k_{\mathrm{rad}}^{\infty}$ and $k_{\mathrm{nr}}$ are the radiative decay rate in an homogenous unbounded medium and the nonradiative decay rate of the emitter, respectively. A knowledge of the quantum-efficiency is vital for applications requiring a single photon source on demand [1,2] and for tackling challenges such as strong light-atom interaction or long distance entanglement protocols by means of integrated waveguides and microcavities in solid state systems $[3,4]$. Furthermore, to design optimal optical structures (e.g., nanocavities or plasmonic), which enhance the collection efficiency or modifies the radiative or/and nonradiative decay paths [3-7], a precise measurement of QE is necessary to accurately quantify any improvement obtained in the detected photons from the coupling. Imaging the transition dipole of quantum emitters, and the verification of the dependence of radiative emission properties on the environment, have wide ranging implications in microscopy and biological sensing [8].

Direct measurements of $\mathrm{QE}$ are challenging and require a priori knowledge of the emission dipole orientation, and a separate measurement of the radiative and nonradiative decay rates. To determine the dipole orientation of single molecules and colloidal quantum dots, methods such as defocused [9,10], direct imaging [11], or near field microscopy [12-14] have been successfully demonstrated. This is achieved by imaging the emission pattern of the collected photons with a high numerical aperture objective in the back focal plane. To extract information about radiative and nonradiative decay rates for an emitter close to the material-air interface, one can modify the local dielectric environment of the emitters. This is enabled by adding to the emitter environment a medium with a matching [15] or a different refractive index [16]. Using such approaches, radiative and nonradiative decay rates of single molecules and quantum dots were separately obtained. In an alternative method, a scanning metal mirror was brought close to a single molecular dipole and its $\mathrm{QE}$ was then measured [17].

Because of the challenge in applying similar strategies to match the refractive index of diamond, we use the novel approach of modifying the environment by creating the color centers at different depths in the same material. We circumvent extra optical interfaces induced by growing diamond on diamond and/or additional impurities or charges that could change the state of the center after deposition.

Recently, high brightness single photon emitters originating from chromium impurities in diamond were fabricated $[18,19]$. The optical properties of these centers reveal some outstanding features compared to other centers in diamond, such as very narrow spectral emission at room temperature (a few nanometers), short excited state lifetime of $<3.5 \mathrm{~ns}$, and large dipole moment. These attributes make them leading candidates for applications in quantum information science [1], subdiffraction microscopy [20], and biological systems [21].

In this Letter we implement ion implantation and imaging techniques to measure directly the quantum efficiency of single centers in monolithic diamond. Employing this approach, we first imaged the emission patterns of single chromium emitters to identify their emission dipole orientation. We then measured the decay rates from emitters located in close proximity to the diamond surface and from emitters located deep in the diamond crystal. The information obtained enabled the determination of the quantum efficiency of individual single photon emitters.

To fabricate the emitters close to the diamond-air interface, chromium ions (fluence of $10^{10}$ ions $/ \mathrm{cm}^{2}$ ) and oxygen ions (fluence of $1.5 \times 10^{10}$ ions $/ \mathrm{cm}^{2}$ ) were accelerated to $50 \mathrm{keV}$ and $19.5 \mathrm{keV}$, respectively, and implanted into a (100) oriented type IIA diamond $([N]<$ $1 \mathrm{ppm},[B]<0.05 \mathrm{ppm})$. To modify the dielectric environment of the emitters, chromium and oxygen were implanted into the same type of diamond using the same fluencies and an acceleration voltage of 6 and $3.6 \mathrm{MeV}$, respectively. 
The samples were then annealed to $1000^{\circ} \mathrm{C}$ for $2 \mathrm{~h}$ under a forming gas $\left(95 \% \mathrm{Ar}-5 \% \mathrm{H}_{2}\right)$ ambient. Figure 1 (a) shows the simulation of the implantations using stopping range of ions in matter (SRIM). From the simulation it is evident that the projected range of the shallow implantation $(50 \mathrm{keV})$ is approximately $25 \mathrm{~nm}$ below the diamond surface, while the projected range of the deep implantation $(6 \mathrm{MeV})$ is $1.5 \mu \mathrm{m}$ below the diamond surface. Note that the annealing step applied after the implantation is not sufficient to cause any diffusion of the $\mathrm{Cr}$ atoms and the end of range of the two implanted chromium ions does not overlap.

The samples were scanned using a confocal microscope as depicted schematically in Fig. 1(b). Single photon emitters were first identified using a Hanbury BrownTwiss (HBT) interferometer and their photoluminescence (PL) spectra were recorded, as shown in Fig. 1(c). The typical PL emission occurs in the range of 748-760 nm. Figure 1(c) clearly demonstrates that emitters with the same zero phonon line (ZPL) can be fabricated by either shallow or deep ion implantation. A second detection channel was added after the dichroic mirror in the confocal setup to image the transition dipole of individual single photon emitters by an imaging lens and a cooled CCD camera with quantum efficiency of $40 \%$ at $750 \mathrm{~nm}$.
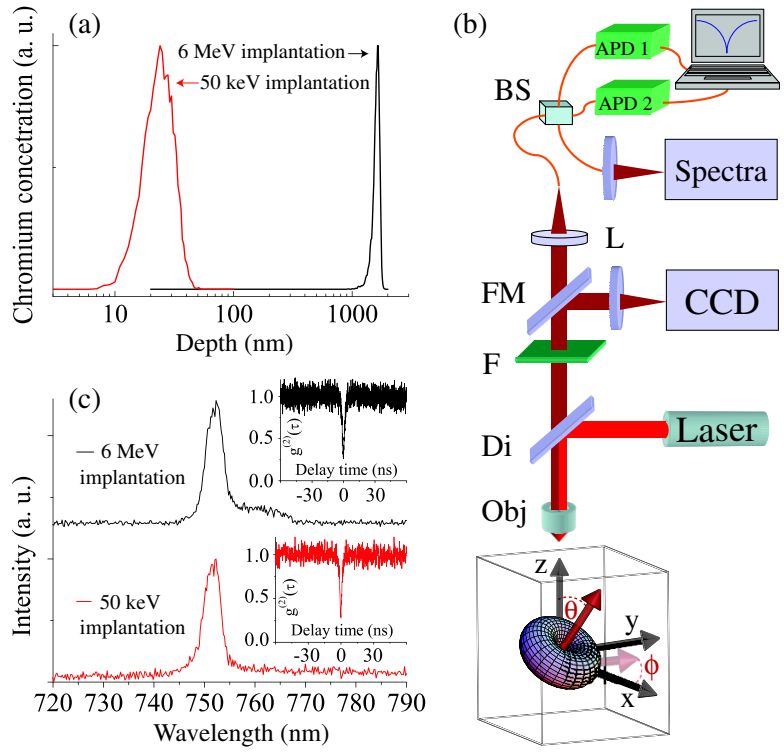

FIG. 1 (color online). (a) Concentration profiles of implanted chromium ions into diamond using an acceleration voltage of $50 \mathrm{keV}$ (red curve) and $6 \mathrm{MeV}$ (black curve) determined using SRIM. (b) Schematic illustration of the confocal microscope (Obj $100 \times 0.95$ numerical aperture objective; Di dichroic mirror; F bandpass filter, FM flip mirror; BS beam splitter, $\mathrm{APD}_{1,2}$ single photon detectors), with a CCD imaging channel. An illustration of the emitting dipole orientation with respect to the diamond sample and the optical axis $(z)$ of the objective, identified by the polar angle $\theta$ and the azimuth angle $\Phi$. (c) Example of PL spectra of chromium related centers with the same zero phonon line created by ion implantation using energies of $50 \mathrm{keV}$ (red curve) and a $6 \mathrm{MeV}$ (black curve). The insets show antibunching curves demonstrating single photon emission.
In the first part of the experiment we imaged the emission dipole orientation by recording the angular intensity distribution of single emitters in the back focal plane of a high numerical aperture objective using a CCD camera. Figure 2(a) shows a typical objective back-focal-plane dipole image recorded from a single chromium center in bulk diamond, that differs significantly from a standard
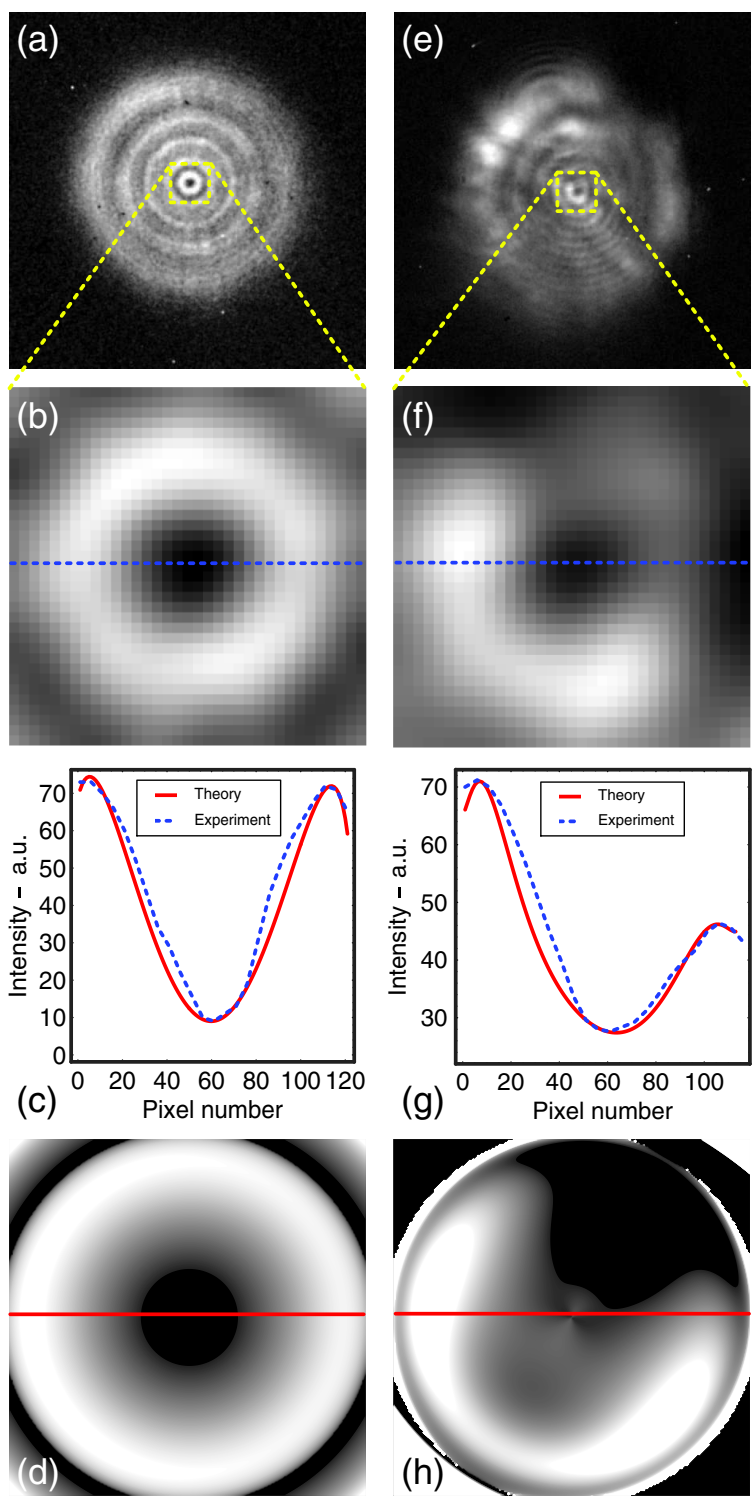

FIG. 2 (color online). (a),(e) Two images of the intensity distribution of the emission dipole, from chromium single photon emitters (ZPL at $753 \mathrm{~nm}$ ) created in bulk diamond and in submicron diamond crystal, respectively. Integration times were 200 and $60 \mathrm{~s}$, respectively. (b),(f) Magnified area of the central ring of the images depicted in (a),(e). (c),(g) The cross section experimental data and the theoretical fit of the emission pattern are shown in (b),(f). The polar angles of the emitters are $\theta=$ $(1 \pm 1)^{\circ}$ and $\theta=(49 \pm 2)^{\circ}$ for the bulk and submicron diamond, respectively, while the azimuth angles are $\phi=(0 \pm 5)^{\circ}$ and $\phi=(69 \pm 2)^{\circ}$.(d),(h) Calculated pattern of the dipole emission shown in (b),(f) using the parameters from the fit. 
Airy point spread function. Concentric doughnut-shaped rings, associated with being imaged through the aperture of a dry objective, are observed in the CCD image [22]. The uniform intensity distribution of the bright rings with dark centers indicates that the emitter is oriented nearly orthogonal to the diamond-air interface. This confirms that the $\mathrm{Cr}$ center is not aligned along a $\langle 111\rangle$ direction neighboring a vacancy, as then a trigonal symmetry is expected. More than 20 single emitters were imaged individually, all confirming a very similar dipole orientations, with polar angles between 0 and $2^{\circ}$, which are within our method sensitivity.

For the sake of comparison, Fig. 2(e) shows a typical objective back-focal plane dipole image of the $\mathrm{Cr}$ centers created in submicron diamond ( $300 \mathrm{~nm}$ average size), with the same ZPL as the emitter shown in Fig. 2(a). In this case, as expected, the emission dipole orientation changes from crystal to crystal and it is clearly not parallel to the optics axis. Figures 2(b) and 2(f) show a magnified area of the central rings of the images shown in (a), (e), respectively. Figures 2(c) and 2(g) show the cross section data and the fit of the emission pattern shown in Figs. 2(b) and 2(f), respectively, according to the theory presented in [11]. From the fit, the dipole polar coordinate $\theta$ was estimated to be less than $(1 \pm 1)^{\circ}$ and the azimuth angle $\Phi=(0 \pm 5)^{\circ}$ for the bulk diamond. While for this particular nanocrystal, $\theta=(49 \pm 2)^{\circ}$ and $\Phi=(69 \pm 2)^{\circ}$. Figures 2(d) and 2(h) show a two-dimensional calculated pattern of the dipole emission shown in Figs. 2(b) and 2(f) using the parameters from the fit. Excellent agreement between the theory and experiment is obtained, demonstrating that the technique can be successfully applied to color centers in bulk and nanodiamonds to fully determine their 3D orientation.

In our previous work [19], it was determined that the absorption dipole for $\mathrm{Cr}$ centers in bulk diamond is aligned along one of the main crystallographic axes on the plane of the surface and the emitted light is not linearly polarized. The nearly orthogonal emission dipole observed in this work elucidates that the emission dipole of the chromium centers in bulk diamond is nearly perpendicular to its absorption dipole.

In the second part of our experiment, we measured the total excited state lifetime and the QE of individual emitters in bulk diamond. In the situation of a linear dipole located at a distance $d<\lambda$ from a dielectric interface, Lukosz and Kunz [23] showed that the radiative decay rate $\left(k_{\mathrm{rad}}\right)$ depends on the distance $d$, the refractive index of each dielectric medium and the excitation dipole orientation polar angle $\theta$, with respect to the normal to the interface.

We denote by $k_{\infty}=k_{\mathrm{nr}}+k_{\mathrm{rad}}^{\infty}$, the total decay rate of an emitter in an unbounded homogeneous medium and $\alpha\left(d, \theta, n_{1}\right)=k_{\text {rad }}\left(d, \theta, n_{1}\right) / k_{\text {rad }}^{\infty}$ the modification of the decay rate in the presence of the dielectric interface, where $n_{1}=n_{2} / n$, being $n_{2}$ the index of refraction of the medium after the interface. The physical interpretation can be qualitatively described by classical electrodynamics. When the dipole radiates, its field is partly reflected by the interface. The dipole can then interact with its own field. This self-interaction modifies the oscillation amplitude (and frequency) of the dipole and, as a consequence, affects its radiative decay time. The total decay rate for a linear dipole can thus be generally written as

$k(d, \theta)=k_{\mathrm{nr}}+k_{\mathrm{rad}}^{\infty}\left[\alpha\left(d, n_{1}\right)_{\|} \sin ^{2}(\theta)+\alpha\left(d, n_{1}\right)_{\perp} \cos ^{2}(\theta)\right]$,

where $\alpha_{\|, \perp}$ refers to a parallel and orthogonal dipole to the interface, with the algebraic expression given in [23]. If an emitter is moved far from the interface or the refractive index difference of the interface is reduced to zero both $\alpha_{\|, \perp}=1$, and the excited state lifetime is independent of the dipole orientation. From the decay rates for dipoles close to an interface and in an unbounded medium, we deduce the value $\beta=k\left(d, \theta_{e}\right) / k_{\infty}$. The $\mathrm{QE}$ can thus be rewritten as $\mathrm{QE}=(1-\beta) /\left[1-\alpha\left(d, \theta, n_{1}\right)\right]$ [24].

In our particular case the chromium centers implanted with energies of $6 \mathrm{MeV}$ are considered to be in an unbounded medium $(d \simeq 1.5 \mu \mathrm{m}>\lambda)$ and far from the diamond-air boundary, while centers created using a $50 \mathrm{keV}$ implantation are located near a dielectric interface. Therefore, measuring the excited state lifetime of deep implants will provide direct information of $k_{\infty}$, while measuring the decay rates of chromium centers engineered near the surface will allow us to deduce $k(d, \theta)$. To exclude any wavelength dependent effect, only emitters with the same peak emission are compared. Note that since the centers are embedded in the diamond matrix, the immediate surroundings in both the shallow and the deep implantations are the same and therefore $k_{\mathrm{nr}}$ can be assumed to remain constant [24].

Figures 3(a) and 3(b) show the fluorescence decay rates of two single chromium emitters for both shallow and deep implantations. The angle $\theta$ of the emission dipole of these emitters was found to be $\theta=(1 \pm 1)^{\circ}$ [Fig. 2(a)] and $\theta=(0.5 \pm 1)^{\circ}$ (not shown here), respectively. The data
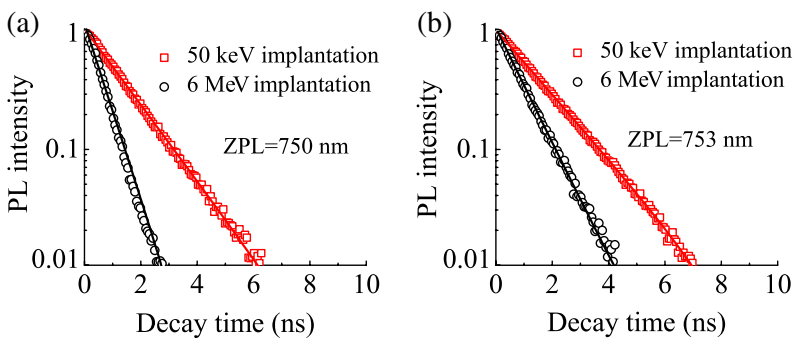

FIG. 3 (color online). (a) Direct lifetime measurement of a single emitter with a ZPL centered at $750 \mathrm{~nm}$ close to the diamond-air interface (squares) and of an emitter with the same ZPL located $1.5 \mu \mathrm{m}$ below the diamond surface (circles). The data were fit using a single exponential fit (solid line). (b) Decay rate measurements recorded from a different emitter with a ZPL centered at $753 \mathrm{~nm}$ located near the interface (squares) and deep in the diamond crystal (circles). 


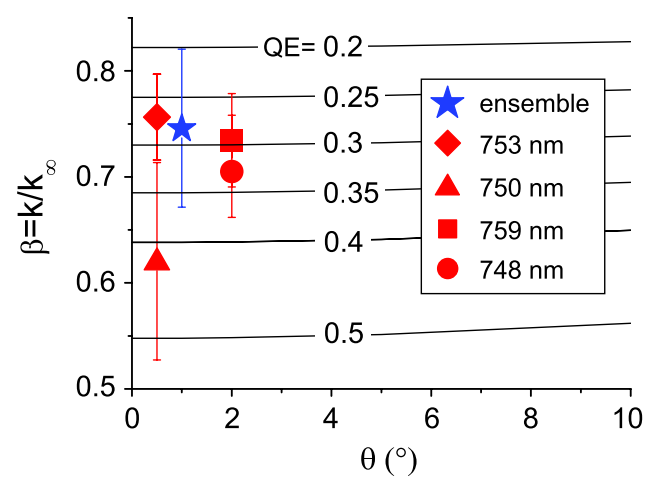

FIG. 4 (color online). Orientational dependence of $\beta$ on the polar angle. The solid black lines correspond to calculated values for different values of $\mathrm{QE}$ and with $d=25 \pm 5 \mathrm{~nm}$. The measured values of $\beta$ from an ensemble measurement (blue star) and several single centers with different ZPL (red circle, square, triangle, and diamond) are superimposed.

were fitted by using a monoexponential curve with a relative uncertainty of $0.5 \%$. The reduction of the total decay rate for emitters located near the diamond-air interface is clearly seen from these measurements. From the measured decay rates for the shallow and the deep implantations, the value $\beta=k(d, \theta) / k_{\infty}$ was deduced, as an average obtained for emitters with the same ZPL. For two single emitters at 750 and $753 \mathrm{~nm}$, the calculated parameter $\alpha$ and the measured value of $\beta$ yield a $\mathrm{QE}=$ $0.42 \pm 0.06$ and $\mathrm{QE}=0.29 \pm 0.05$, respectively. To the best of our knowledge, this is the first direct measurement of $\mathrm{QE}$ of a single color center in diamond.

In the last part of our experiment, we measured the average QE of an ensemble of single chromium emitters in bulk diamond, regardless of their peak emission wavelength. The average excited state lifetime for the centers located near the diamond-air interface is $1.24 \pm 0.13 \mathrm{~ns}$, while the lifetime of the centers located deep in the diamond crystal is $0.92 \pm 0.09 \mathrm{~ns}$. A clear reduction of the excited state lifetime for the emitters located in an unbounded medium is noticeable also in an ensemble measurement. This result confirms that the centers are associated with a linear dipole since a 2D dipole orthogonal to the surface would not provide such a variation in the measured excited state lifetime in the shallow and deep implantation [24].

The QE was computed for various polar angles and values of $\beta$ and is plotted in Fig. 4 (black lines). The nearly orthogonal emission dipole observed for the centers yields an ensemble value of $\alpha=0.098$ [23] and results in an averaged $\mathrm{QE}=0.28 \pm 0.04$ for chromium emitters, regardless their peak emission wavelength. The experimental values of the $\mathrm{QE}$ of several single centers with the same ZPL and of the ensemble measurement are shown in Fig. 4. $\mathrm{A} Q \mathrm{QE}$ in the range of $30 \%$ can be associated to the presence of a metastable state or to additional nonradiative process such as decay through phonons, ionization, or heat, which strongly depend on the environment. The intersystem crossing rate for the chromium emitters is $k_{\mathrm{ISC}}=$ 5.1 MHz, as was deduced from analyzing the second order autocorrelation function. This value indicates that the population of the metastable state does not significantly reduce the value of the $\mathrm{QE}$ since the radiative decay is $\sim 313 \mathrm{MHz}$ and the nonradiative decay $\sim 769 \mathrm{MHz}$.

To summarize, we directly measure the quantum efficiency of single chromium emitters in bulk diamond and present their emission dipole images. The peculiarity of an emission dipole nearly always orthogonal to the bulk diamond surface is particularly advantageous for integration of these emitters with cavities or diamond nanoantennas [25]. We envisage that a similar approach could be used to determine the actual quantum efficiency of $\mathrm{NV}^{-}$, which is commonly inferred on the basis of circumstantial evidence [26].

The Australian National University is acknowledged for providing ion implanting facilities. This work was supported by the Australian DIISR, ARC, and the European Union under EQUIND IST-034368.

*sacas@unimelb.edu.au

iah@unimelb.edu.au

[1] J. L. O'Brien et al., Nat. Photon. 3, 687 (2009).

[2] J. Y. Cheung et al., J. Mod. Opt. 54, 373 (2007).

[3] P. E. Barclay et al., Appl. Phys. Lett. 95, 191115 (2009).

[4] S. Schietinger et al., Nano Lett. 9, 1694 (2009).

[5] A. Kinkhabwala et al., Nat. Photon. 3, 654 (2009).

[6] O. L. Muskens et al., Nano Lett. 7, 2871 (2007).

[7] C. Vion et al., Opt. Express 18, 7440 (2010).

[8] E. Toprak et al., Proc. Natl. Acad. Sci. U.S.A. 103, 6495 (2006).

[9] M. Bohmer et al., J. Opt. Soc. Am. B 20, 554 (2003).

[10] D. Patra et al., Appl. Phys. Lett. 87, 101103 (2005).

[11] M. A. Lieb et al., J. Opt. Soc. Am. B 21, 1210 (2004).

[12] E. Betzig et al., Science 251, 1468 (1991).

[13] N. F. van Hulst et al., J. Chem. Phys. 112, 7799 (2000).

[14] Drezet et al., J. Lumin. 107, 176 (2004).

[15] X. Brokmann et al., Phys. Rev. Lett. 93, 107403 (2004).

[16] J. Macklin et al., Science 272, 255 (1996).

[17] B. C. Buchler et al., Phys. Rev. Lett. 95, 063003 (2005).

[18] I. Aharonovich et al., Nano Lett. 9, 3191 (2009).

[19] I. Aharonovich et al., Phys. Rev. B 81, 121201(R) (2010).

[20] E. Rittweger et al., Nat. Photon. 3, 144 (2009).

[21] C.-C. Fu et al., Proc. Natl. Acad. Sci. U.S.A. 104, 727 (2007).

[22] R. M. Dickson, D. J. Norris, and W. E. Moerner, Phys. Rev. Lett. 81, 5322 (1998).

[23] W. Lukosz and R. Kunz, J. Opt. Soc. Am. 67, 1607 (1977).

[24] X. Brokmann et al., Chem. Phys. 318, 91 (2005).

[25] T. M. Babinec et al., Nature Nanotech. 5, 195 (2010).

[26] A. T. Collins et al., J. Phys. C 16, 2177 (1983). 